\title{
Locomotor versatility in the white-handed gibbon (Hylobates lar): A spatiotemporal analysis of the bipedal, tripedal, and quadrupedal gaits
}

\author{
Evie E. Vereecke ${ }^{\mathrm{a}, *}$, Kristiaan D’Août ${ }^{\mathrm{a}, \mathrm{b}}$, Peter Aerts ${ }^{\mathrm{a}, \mathrm{c}}$ \\ ${ }^{a}$ Laboratory for Functional Morphology, University of Antwerp, Universiteitsplein 1 B-2610 Wilrijk, Antwerp, Belgium \\ ${ }^{\mathrm{b}}$ Centre for Research and Conservation, Belgium \\ ${ }^{c}$ Department of Movement and Sports Sciences, University of Ghent, Ghent, Belgium
}

Received 17 May 2005; accepted 23 December 2005

\begin{abstract}
This study gives a qualitative and quantitative description of the different terrestrial locomotor modes of a group of white-handed gibbons (Hylobates lar) from the Wild Animal Park Planckendael, Belgium. The gibbons were filmed during voluntary locomotion on a grassy and smooth substrate and on a pole. These video images allowed us to define seven different gait types, based on spatial and temporal footfall patterns. Consequent digitization of the video images $(n=254)$ yielded duty factors, stride lengths, and stride frequencies of the fore- and hind limbs during locomotion at a wide range of speeds. These spatiotemporal gait characteristics were regressed against velocity, and the regression lines of the different gait types were compared. In addition, gibbon bipedalism was compared with bonobo (Pan paniscus) and human bipedalism. Gibbons appear to be very versatile animals, using a bipedal, tripedal, or quadrupedal gait during terrestrial travel with an overlapping speed range. The spatiotemporal characteristics of these gaits are largely similar, although they have clearly distinct footfall patterns. Bipedal walking on the pole is slightly different from terrestrial bipedalism, but differences between substrate types (grass vs. catwalk) are subtle. During bipedalism, gibbons increase both stride length and frequency to increase speed, just as humans and bonobos do, but at a given speed, gibbons take relatively larger strides at lower rates. Bipedal walking in gibbons also appears to be relatively fast-gibbons could keep on walking at speeds where humans have to start running. Apparently, adaptations for arboreal locomotion have not constrained the terrestrial locomotor abilities of gibbons. This may indicate that the step from an arboreal ancestral ape to a terrestrial, upright bipedal hominin might not be difficult and that structural specializations are not a prerequisite for adopting a (non-habitual) bipedal gait.
\end{abstract}

(C) 2006 Elsevier Ltd. All rights reserved.

Keywords: Velocity; Terrestrial locomotion; Footfall patterns; Evolution of bipedalism

\section{Introduction}

Gibbons are highly arboreal primates that travel through the forest canopy primarily by arm-swinging or brachiating, but

\footnotetext{
Grant sponsorship: Research assistant and research project (G.0209.99N) of the Fund for Scientific Research, Flanders (Belgium).

* Corresponding author. Laboratory for Functional Morphology, Department of Biology, University of Antwerp, Universiteitsplein 1 B-2610 Wilrijk, Antwerp, Belgium. Tel.: +32 3820 2260; fax: +32 38202271 .

E-mail addresses: evie.vereecke@ua.ac.be (E.E. Vereecke), kristiaan.daout@ ua.ac.be (K. D’Août), peter.aerts@ua.ac.be (P. Aerts).
}

they also employ a wide variety of other locomotor modes, such as diving, leaping, bridging, bipedal walking, running, quadrumanous climbing, and scrambling (Carpenter, 1964; Ellefson, 1974; Baldwin and Teleki, 1976; Fleagle, 1976; Gittins, 1983; Hollihn, 1984; Tuttle, 1986; Cannon and Leighton, 1994; Sati and Alfred, 2002; Vereecke et al., 2005a). Yet, while they are only observed occasionally on the ground in the wild (when crossing roads or gaps in fragmented forests; Sati and Alfred, 2002; Rawson, pers. comm.; Thampy, pers. comm.), gibbons in captivity prove to be equally versatile in their terrestrial locomotor behavior. They make use of several bipedal, tripedal, or quadrupedal 
gaits and switch smoothly from one type to the other when moving around (Carpenter, 1964; Baldwin and Teleki, 1976; Fleagle, 1976; Nakano, 2002; pers. obs.). Obviously, arboreal adaptations for brachiation (e.g., the elongation of the forelimbs, hands, and feet; Schultz, 1973) have not constrained their terrestrial locomotor abilities.

Nevertheless, except for brachiation (Andrew and Groves, 1976; Jungers and Stern, 1984; Preuschoft and Demes, 1984; Bertram and Chang, 1996; Bertram et al., 1999; Chang et al., 2000; Bertram and Chang, 2001; Usherwood and Bertram, 2003; Bertram, 2004) and bipedal locomotion (Carpenter, 1964; Prost, 1967; Tuttle, 1972; Fleagle, 1976; Ishida et al., 1976; Okada et al., 1983; Ishida et al., 1984; Yamazaki and Ishida, 1984; Schmid and Piaget, 1994; Vereecke et al., 2005b), quantitative gait data for gibbons are lacking. Recently, efforts have been made to compile a sound quantitative data set of gait variables for great apes (Aerts et al., 2000; D'Août et al., 2002; Isler and Thorpe, 2003; Isler, 2005; Vereecke et al., 2003, 2004). The lack of information for gibbon locomotion is unfortunate because insights into their locomotor capacities could add to our understanding of hominoid locomotion and perhaps the evolution of habitual terrestrial bipedalism in humans.

As a first step toward filling this gap, the present study aimed to complement the comprehensive and detailed, yet qualitative, description of the types of locomotion observed in a group of captive lar gibbons (Hall's Island, Bermuda) by Baldwin and Teleki (1976). This goal was accomplished by defining some additional gait types and by collecting quantitative data on the spatial and temporal footfall patterns of the different terrestrial locomotor modes of the white-handed gibbon (Hylobates lar) as a function of locomotor speed. In addition, the spatiotemporal gait variables of terrestrial bipedalism (on a grassy and smooth surface) were compared with arboreal bipedalism (on a horizontal pole). We compared bipedalism on the grassy and smooth surfaces to test if gait variables collected in laboratory conditions (smooth walkway) corresponded to those collected on a more natural substrate (grass). Finally, bipedal gait characteristics of gibbons were compared to those of bonobos (Pan paniscus) and humans (Homo sapiens). These species are interesting for comparison, in view of their different morphology, their ability to walk bipedally, and their close phylogenetic relationship with gibbons (McHenry and Corruccini, 1981; Goodman, 1999; Aerts et al., 2000; Vereecke et al., 2003; D'Août et al., 2004; Goodman et al., 2005).

\section{Materials and methods}

For this study, we recorded the bipedal, tripedal, and quadrupedal locomotion of five white-handed gibbons (Hylobates lar) in the Wild Animal Park Planckendael, Belgium (Table 1). The bipedal sequences were recorded under three different conditions: (1) on a horizontal pole, (2) on a mowed grassy substrate, and (3) on a walkway (smooth surface). In the first two settings, we filmed the gibbons on their outdoor island, which is a relatively smooth terrain, mainly covered with
Table 1

Subject data

Subject Year of birth Sex ${ }^{\mathrm{a}}$ Leg length (m) Number of sequences ${ }^{\mathrm{b}}$

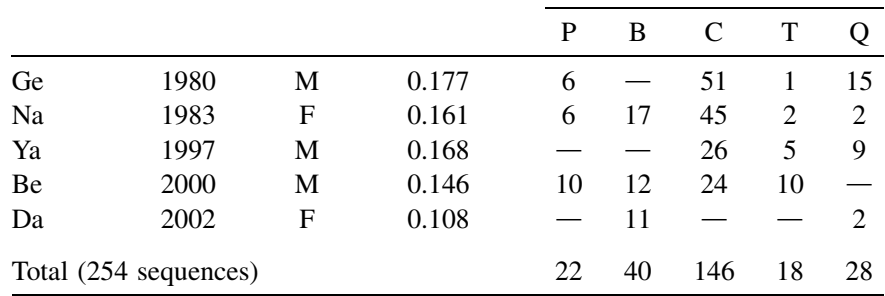

${ }^{\mathrm{a}} \mathrm{F}=$ female, $\mathrm{M}=$ male

${ }^{\mathrm{b}} \mathrm{B}=$ bipedal on grass, $\mathrm{C}=$ bipedal on catwalk, $\mathrm{P}=$ bipedal on pole, $\mathrm{T}=$ tripedal, and $\mathrm{Q}=$ quadrupedal.

grass, but which also contains some trees, shrubs, and wooden climbing structures. In the first setting, the gibbons were filmed during bipedal walking on a 4-m-horizontal wooden pole $(n=22$; Table 1$)$. The pole was positioned $1 \mathrm{~m}$ above the ground (on the island) and had a diameter of $0.15 \mathrm{~m}$. The bipedal sequences in the second setting included only terrestrial bouts $(n=40$; Table 1$)$. The third setting included terrestrial bipedal sequences, but these were recorded in our experimental indoor set-up $(n=146$; Table 1$)$. This set-up consisted of a 4-m-long wooden walkway covered with a non-skid rubber sheet and surrounded by a lattice corridor. More details about this "catwalk" set-up are given in Vereecke et al. (2005b). The tripedal $(n=21)$ and quadrupedal sequences $(n=28$; Table 1$)$ were terrestrial and were recorded on the outdoor island.

The sequences on the outdoor island were filmed from a fixed position, using a digital 8 Sony camera (PAL 50Hz). To allow scaling of the recorded video images, we constructed a grid of $24 \mathrm{~m}^{2}$ on the island, consisting of small wooden sticks, which were put in the ground at $2 \mathrm{~m}$ intervals. This reference grid was only used for the terrestrial sequences. To scale the bipedal sequences on the pole, we put clearly visible marks on the pole at $1 \mathrm{~m}$ intervals. The animals were free to move on the island, in any direction, at any speed, and using whatever locomotor mode they chose. However, in order to standardize the recorded sequences, only the sequences with a reasonably constant velocity and with direction of progression perpendicular to the position of the camera were selected for analysis (visual evaluation). The latter condition was always fulfilled for pole-walking. The sequences from the indoor set-up were spontaneous locomotor bouts, but a lattice corridor confined the direction of progression.

Next, all retained video sequences were played back frame by frame and analyzed by digitizing each limb contact and each limb lift-off with a NAC XY coordinator and by registering the corresponding time code. For each sequence, we measured the following parameters for the left or right fore- and hind limb: stride duration (SD, the time between contact of a limb to the next contact of the same limb), contact time (CT, the absolute duration of time the limb was on the substrate), duty factor (DF, the fraction of the stride duration for which the limb was on the substrate), stride length (SL, the distance traveled during one stride), and stride frequency 
(SF, the number of strides per second), together with the corresponding velocity ( $\mathrm{V}$; see also Table 2 ). The relative phase of a limb was defined as the time at which a limb touched down, expressed as a fraction of the stride duration of the reference limb (see below). These definitions are based on the work of Hildebrand (1967) and Alexander $(1977,1982)$ and are commonly used to describe gait patterns. However, due to the particularity of tripedal skipping (see below), we had to apply a modified definition for the hind limb cycle in the statistical analyses. As described for humans by Minetti (1998), the leading limb alternates from stride to stride during (bipedal) bilateral skipping, resulting in an alternation of long and short stride durations and lengths according to the definitions given above. This alternation also occurs in the tripedal (bilateral) skip of gibbons. Therefore, we redefined the hind limb stride as the gait cycle of the leading hind limb to allow grouping of the tripedal skipping and galloping sequences and to enable comparison with the other gait types. The stride duration was then defined as the time between touchdown of the leading hind limb to the next touchdown of the leading hind limb, which is the contralateral hind limb in the case of tripedal skipping. Stride length and stride frequency were then defined accordingly. These modified definitions do not affect the bipedal and quadrupedal gaits, as the leading hind limb does not alternate with each gait cycle-it is always the same limb that touches down first-in these gaits.

To allow for comparison of the subjects of different size, we treated the square root of the Froude number as dimensionless velocity (DV), and the SL and SF of the hind limbs were scaled to lower leg length using the formulae provided in Table 2 (Alexander and Jayes, 1983; Aerts et al., 2000). As direct leg length measures were not available and were not allowed under the zoo protocol, we had to estimate them from the video images. Lower leg length was measured from knee to ankle and was used instead of total hind limb length since it proved to be the most accurate length measure that can be obtained from the video images (Aerts et al., 2000). Lower leg length is proportional to total hind limb length and has proven to be a good dimension for normalization of the variables (Isler and Thorpe, 2003; Alexander, 2004).

Statistical analyses were executed to test for differences between the different gait types and to investigate the relationship between the spatiotemporal parameters and the velocity within each gait type. To allow for comparison of the bipedal, tripedal, and quadrupedal gaits, only the spatiotemporal

Table 2

Spatiotemporal gait characteristics

\begin{tabular}{lll}
\hline Abbreviation & Parameter & Formula $^{\mathrm{a}}$ \\
\hline $\mathrm{CT}$ & Contact time (s) & \\
$\mathrm{SD}$ & Stride duration $(\mathrm{s})$ & \\
$\mathrm{SF}$ & Stride frequency $(\mathrm{Hz})$ & $1 / \mathrm{SD}$ \\
$\mathrm{DF}$ & Duty factor & $\mathrm{CT} / \mathrm{SD}$ \\
$\mathrm{DSL}$ & Dimensionless stride & $\mathrm{SL} / \mathrm{LL}$ \\
& length (SL) & $\mathrm{SF} \times(\mathrm{LL} / \mathrm{g})^{1 / 2}$ \\
DSF & Dimensionless stride frequency & $\mathrm{V} /(\mathrm{LL} \times \mathrm{g})^{1 / 2}$ \\
DV & Dimensionless velocity $(\mathrm{V})$ &
\end{tabular}

parameters of the hind limbs were included. Linear regressions of the spatiotemporal parameters (dimensionless stride length, DSL; dimensionless stride frequency, DSF; and duty factor, DF) as a function of dimensionless velocity (DV) were calculated in SAS 8.02 for Windows. A mixed linear model (SAS MIXED procedure; SAS Institute, Inc., 1999) was used to calculate the linear equations and to account for within-subject covariability resulting from repeated measures (each animal made multiple bouts and observations of the same subject are usually correlated; Wolfinger and Chang, 1995; Kowalchuk et al., 2004). The Satterthwaite method (Satterthwaite, 1941) was used to adjust the degrees of freedom, which is necessary for repeated measures in unbalanced designs (Schaalje et al., 2001; Kowalchuk et al., 2004). Differences in slope were tested (proc MIXED) and two-by-two comparisons between intercepts of regression lines with statistically similar slopes were executed using a Tukey post-hoc test to correct for multiple comparisons.

For the tripedal $(n=13)$ and quadrupedal $(n=15)$ sequences, we also analyzed the relationship between the spatiotemporal parameters of the forelimb and those of the hind limb. This was accomplished by calculating power functions and Pearson product-moment correlations between the forelimb and hind limb spatiotemporal parameters (CT, SF, SL, and DF). For these calculations, absolute stride lengths (SL) were used instead of dimensionless stride lengths (DSL) because accurate measurements of the forelimb lengths of our gibbons were lacking.

Finally, we included some spatiotemporal data for bipedal walking in bonobos $(n=17)$ and humans $(n=31)$, as presented by Aerts et al. (2000), and calculated linear regression equations for the DSL and DSF as a function of dimensionless walking speed. The human data were obtained from Alexander (1992) and Adachi et al. (1996) and were scaled to lower leg length, assuming a ratio of lower leg length to total hind limb length of 0.54 (Winter, 1991; see also Aerts et al., 2000), to allow for comparison with the gibbon data. We tested for differences in slope and/or intercept between the linear regressions of the three hominoid species using the same linear mixed model as in the analysis of the gibbon data (proc MIXED in SAS 8.02).

\section{Results}

\section{General characteristics and footfall patterns of the different terrestrial gait types of gibbons}

As mentioned in the introduction, the locomotor behavior of captive gibbons has already been described and classified by Baldwin and Teleki (1976). Although they provided a very detailed description of the different gait types, the descriptions were made from an ethological point of view and quantifiable gait parameters are lacking. In order to provide a more quantitative gait description, we made gait diagrams and footfall patterns of each gait type and compared the gait parameters of the different terrestrial gaits. We have tried to follow the terminology of Baldwin and Teleki (1976), which was often impossible 
since some gait types, characterized by distinct footfall patterns, were not observed previously.

Gibbons use several gaits during terrestrial locomotion. In order to classify these different gait types, we have made a "determination key" with corresponding gait diagrams (Fig. 1). In these gait diagrams, the left forelimb-or hind limb in the bipedal gaits - was chosen as reference limb in accordance with the phase diagrams presented in Alexander (1982). A first discrimination was made based on the number of supporting limbs during the gait cycle. During the bipedal gaits, only the hind limbs contact the substrate. There are two symmetrical bipedal gaits, namely, bipedal walking, ${ }^{1}$ with a duty factor greater than 0.5 , and bipedal running, with a duty factor of less than 0.5 ; there is one asymmetrical gait, bipedal galloping (Whitall and Caldwell, 1992; Caldwell and Whitall, 1995; Peck and Turvey, 1997), during which both feet are less than half a cycle out of phase. In the tripedal and quadrupedal gaits, both forelimbs and hind limbs contact the substrate during a gait cycle, but there is a clear visual distinction between both gaits. During tripedalism, the forelimbs are half a cycle out of phase, and the cycle frequency of the hind limbs doubles that of the forelimbs. For each hand contact, there is contact of both the leading and trailing foot. If the leading hind limb does not change from stride to stride, the gait is called a tripedal gallop; if the leading hind limb switches with each stride, the gait is a tripedal (bilateral) skip (cf. Minetti, 1998). During quadrupedalism, the forelimbs are in phase, and two gait subtypes are distinguished by the absence or presence of an aerial phase, during which both hind limbs are off the ground. In a crutching walk there is no hind limb aerial phase, whereas during the half-bound or crutching gallop, there is a hind limb aerial phase.

The spatial and temporal footfall patterns of the different gaits are shown in Figure 2 and are illustrated in Figure 3. A detailed description of each gait type is provided below. Although we classify the locomotion of gibbons into several distinct gait types, gibbons often switch from one gait type to another within one bout and, hence, the different gaits are not always clearly distinct from each other. Moreover, as shown in Figure 4, a clear relationship between transition from one type to another and locomotor speed is not present. The voluntary adopted speeds of the different gaits overlap greatly, and only the tripedal gaits are used at slightly higher speeds in the present study.

\section{Bipedalism}

Bipedal walking is a gait type with a nearly erect, rather immobile trunk and an alternating contact of left and right foot with the substrate. Hip and knee are flexed during the entire

\footnotetext{
${ }^{1}$ We used the spatiotemporal determinant to discern walking and running from each other. We are fully aware of the fact that an alternative discrimination based on the dynamics of the body's center of mass may classify the walking and running trial differently (Farley and Ferris, 1998). We opted for the spatiotemporal classification, as this is readily accessible from simple kinematics and is as valuable and useful as any other rigorous method to clarify and compare gaits.
}

gait cycle (Fig. 3A). The ankle is plantarflexed at the end of the swing phase and touches down with the hallux, followed by midfoot and toe contact, heel touchdown, and a forward transmission of the body weight (Vereecke et al., 2005b).

In bipedal walking, we can distinguish two subtypes, namely, bipedal walking sensu stricto (s.s.) and hand-assisted bipedal walking. During bipedal walking s.s., only the feet contact the substrate, and the entire body weight is supported by both hind limbs. The position of the forelimbs is variable: flexed maximally at elbow and wrist and held immobile against the chest, slightly flexed and held above the shoulder, or hanging loosely beside the trunk. Whatever their position, they never contact the substrate or assist in walking. In contrast, during hand-assisted (HA) bipedal walking, there is contact of one or two hands with the substrate or with overhanging branches. The forelimbs hang loosely beside the trunk and give some assistance to the hind limbs during walking. These HA bipedal bouts were not included in our statistical analyses.

Both types of bipedal walking are used terrestrially and arboreally (e.g., on branches, inclined poles, and ropes; Fig. 5A,D) and at a wide range of speeds $(0.5-2.8 \mathrm{~m} / \mathrm{s}$ in the present recordings; Table 3, Fig. 4). During arboreal bipedalism, which occurs frequently in the wild while walking over large boughs (Baldwin and Teleki, 1976; Fleagle, 1976; Gittins, 1983; Sati and Alfred, 2002), the opposable hallux is used to grasp the branch.

At high velocities ( $>2.8 \mathrm{~m} / \mathrm{s}$ in the present recordings) two other bipedal gaits are used. Bipedal running (Figs. 2, 3B, 5A) is a symmetrical gait, similar to bipedal walking (s.s.), but the duty factor is less than 0.5 . The posture is slightly modified: the trunk is more bent forward, the hip and knee are more strongly flexed, the recovery movements of the forelimbs are more cyclic than during bipedal walking (s.s.), and there is no heel contact. The bipedal gallop (Fig. 2) is an asymmetrical gait characterized by a successive (left-right or right-left) foot contact followed by a short aerial phase. The frontal plane of the trunk is held obliquely with respect to the line of progression and the forelimbs are raised, resembling the bipedal hopping of sifakas (Fleagle, 1988; Minetti, 1998; Wunderlich et al., 2004). Both bipedal running and galloping are rarely used, and therefore, we were unable to collect enough sequences to include them in the spatiotemporal analyses.

\section{Tripedalism}

During tripedalism, one hand is put on the ground and both legs are swung over it to contact the substrate successively (Figs. 1, 3D), which makes this gait clearly distinct from HA bipedalism, in which one or both hands provide only a little assistance to the fully loaded hind limbs. There are never more than three (or less than one) limbs in contact with the substrate at the same time, and during the hind limb aerial phase, there is only support of one forelimb (Fig. 2). Due to this forelimb contact, the trunk is more bent forward than during bipedalism and there are marked movements of both arms and trunk in three dimensions (Fig. 3D). The forelimbs are slightly flexed at the elbow and strongly flexed at the wrist, and they make circular movements prior to hand 


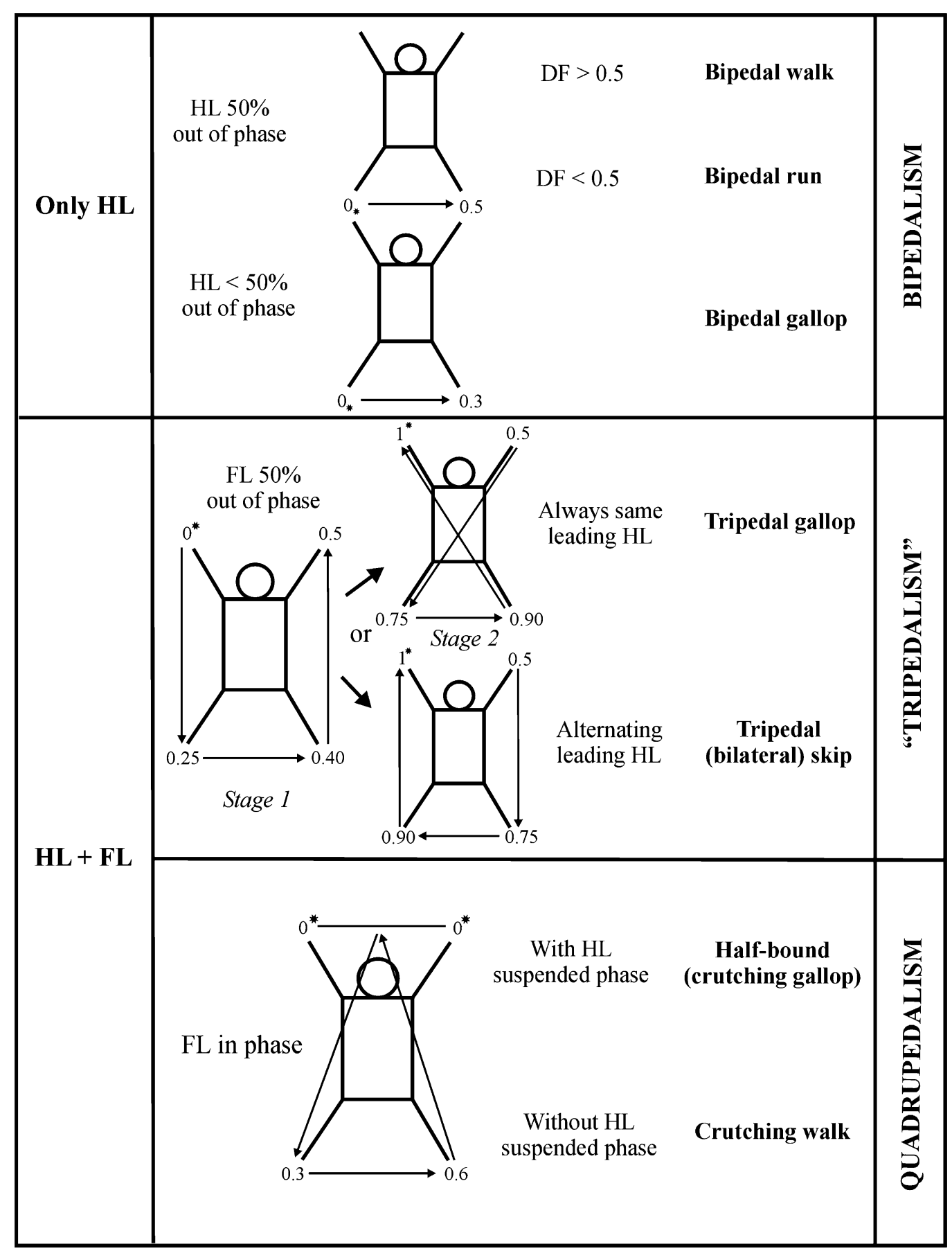

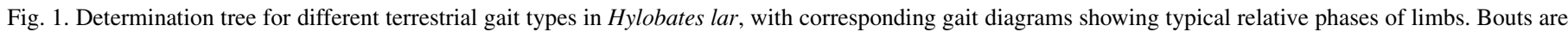

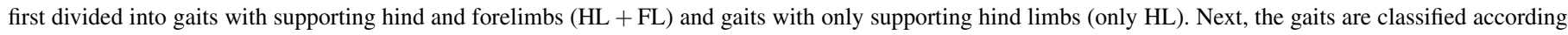

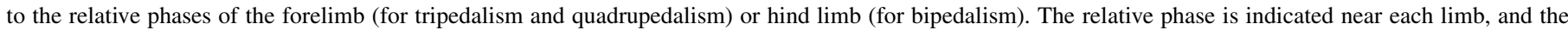

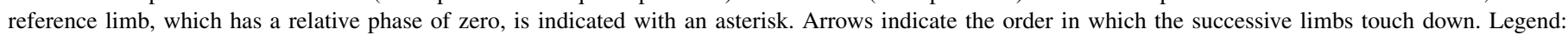
$\mathrm{FL}=$ forelimb, $\mathrm{HL}=$ hind $\operatorname{limb}, \mathrm{DF}=$ duty factor.

contact. There is an alternation of left and right forelimb contact, during which the hand contacts the substrate in various positions, with the knuckles, the dorsal side of the wrist, or the medial side of the hand and pollex. There are two tripedal subtypes, which are both characterized by symmetrical placement of the forelimbs and an asymmetrical placement of the hind limbs in time. In addition, the frequency of the hind limb cycle is always double that of the forelimb cycle.
During the tripedal gallop, the leading hind limb does not change from stride to stride, and thus the feet are always put on the substrate in the same order (e.g., left-right, left-right, left-right, etc.). During tripedal skipping, however, there is an alternation of the leading hind limb with each stride, and the left and right foot contact the substrate in alternating order (e.g., left-right, left-right, left-right, etc.; Figs. 1, 2). 
TEMPORAL

SPATIAL

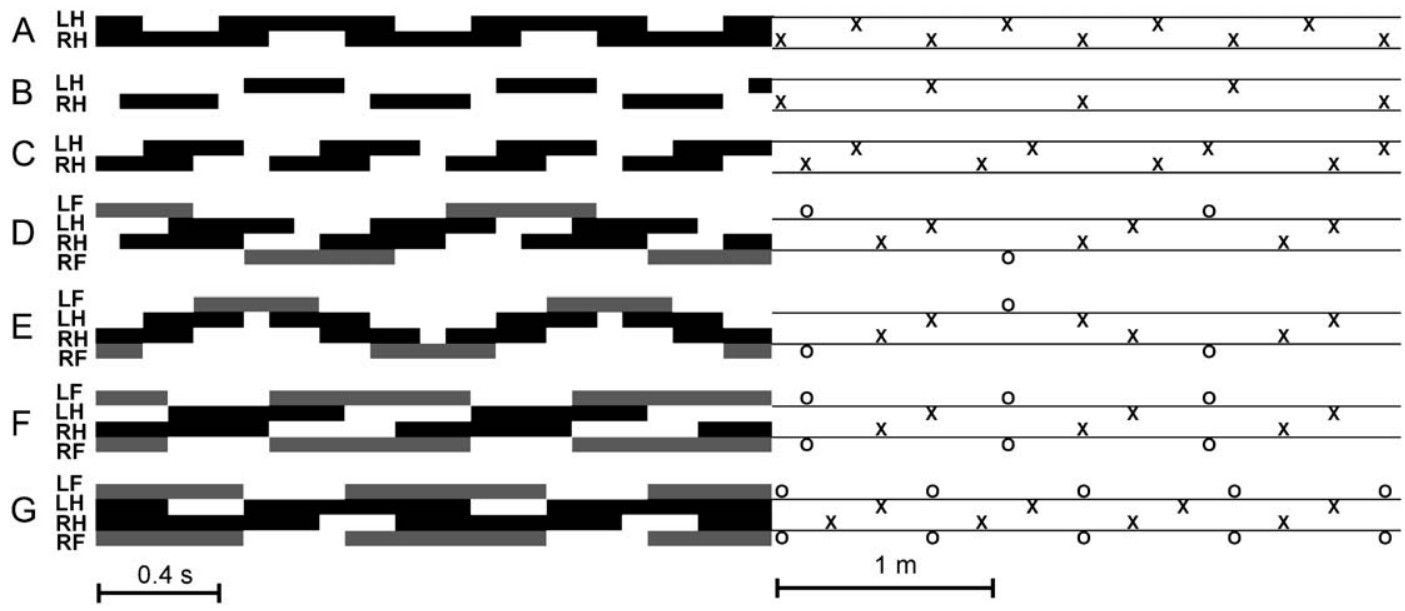

Fig. 2. Temporal (left) and spatial (right) footfall patterns for different gait types in Hylobates lar. Legend: (A) bipedal walk, (B) bipedal run, (C) bipedal gallop, (D) tripedal gallop, (E) tripedal skip, (F) half-bound, and (G) crutching walk; grey bars or $\mathrm{O}=$ hand contact, black bars or $\mathrm{X}=\mathrm{foot}$ contact; $\mathrm{LF}=$ left forelimb, $\mathrm{LH}=$ left hind limb, $\mathrm{RF}=$ right forelimb, $\mathrm{RH}=$ right hind limb.

The tripedal skip is used more frequently than the tripedal gallop and both tripedal gaits were used most commonly (but not exclusively) by the subadult male (Table 1). Tripedalism is performed at rather high speeds $(1-3 \mathrm{~m} / \mathrm{s}$; Table 3, Fig. 4) and is mostly terrestrial, although some tripedal bouts on thick horizontal branches were occasionally observed in captivity (pers. obs.). To our knowledge, no observations of tripedalism have been reported thus far for wild gibbons.

A



B

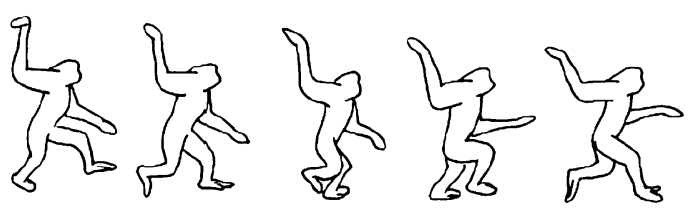

C

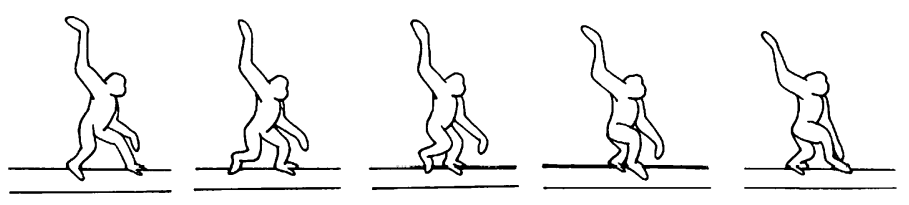

D
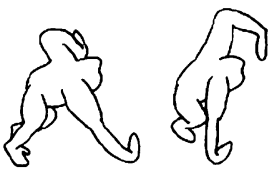

तिक

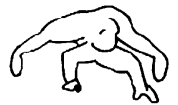<smiles>CC(C)(C)CCc1ccccc1</smiles>

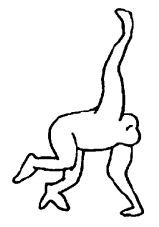



E
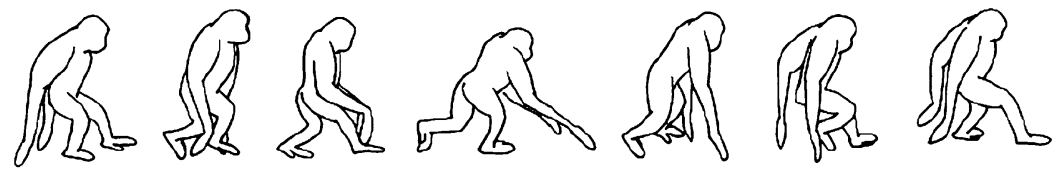

Fig. 3. The different gait types, as drawn from consecutive video images: (A) bipedal walking, (B) bipedal running, (C) bipedal walking on pole, (D) tripedal skipping, and (E) half-bound. 


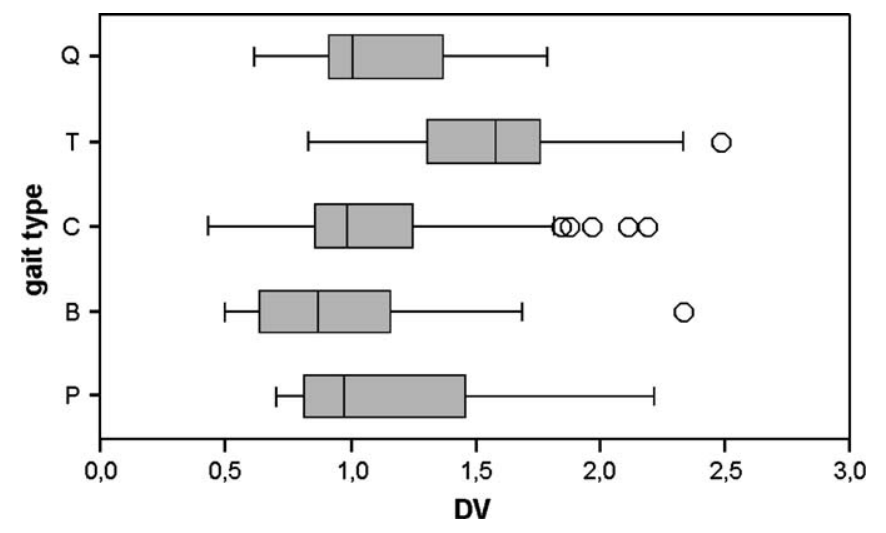

Fig. 4. Boxplots showing the overlapping speed ranges for the different gait types. The boxes illustrate median and standard deviation, the whiskers and circles (i.e., outlier $>1.5$ box length) denote minimum and maximum values of DV. Legend as for Table 1.

\section{Quadrupedalism}

During quadrupedalism, both hands contact the substrate (almost) simultaneously and both hind limbs are swung between them, touching the substrate successively and always in the same order (Figs. 1, 2, 3E; cf. tripedal galloping). The elbow joints are extended during the forward swing of the hind limbs, and thus it resembles walking with crutches, hence the name "crutching walk" given by Baldwin and Teleki (1976). The trunk has a varying inclination of $5^{\circ}$ to $50^{\circ}$ with the vertical during hylobatid quadrupedalism, which is more erect than during bonobo quadrupedalism $\left(>50^{\circ}\right.$; D'Août et al., 2002) due to the relatively longer forelimbs of gibbons. The hands usually make contact between the successive toe-off phases of the left and right foot and leave contact at the moment that the second foot touches the substrate
(Fig. 2). The hands are placed perpendicular to the direction of progression, and the load is supported by the widely abducted pollex and the fingers or on the medial side of the hand and pollex, but never on the knuckles (unlike knucklewalking). A typical quadrupedal sequence can be described as follows: initial contact with left hind limb (LH), hand-off by left (LF) and right forelimb (RF), initial contact with right hind limb $(\mathrm{RH})$, toe-off by LH, initial contact with LF and RF, toe-off by RH, initial contact by LH, etc. Again, two gait subtypes can be distinguished, namely, the crutching walk without a hind limb aerial phase and the half-bound or crutching gallop with a short hind limb aerial phase (Figs. 1, 2). Occasionally, some variations on these general patterns occur (e.g., sequences in which both feet are put on the substrate simultaneously or sequences in which the left and right hand contact the substrate alternately). These variations were not included in the statistical analyses.

Quadrupedalism is used at more moderate speeds (0.7$2.4 \mathrm{~m} / \mathrm{s}$; Table 3, Fig. 4) and is predominantly terrestrial (Fig. 5C). All of the gibbons studied used the quadrupedal gait regularly, but the adult male did so with the highest frequency (Table 1). The half-bound is also more frequently used than the crutching walk. Some half-bound sequences on a horizontal rope ladder were observed in captivity (Fig. 5B) but, to our knowledge, no arboreal crutching walk or half-bound sequences have been observed in the wild.

\section{Spatiotemporal characteristics of the hind limbs during the different gait types}

The mean and standard deviation of the (absolute) spatiotemporal parameters, as well as the minimum and maximum values, are presented for each gait type in Table 3. The

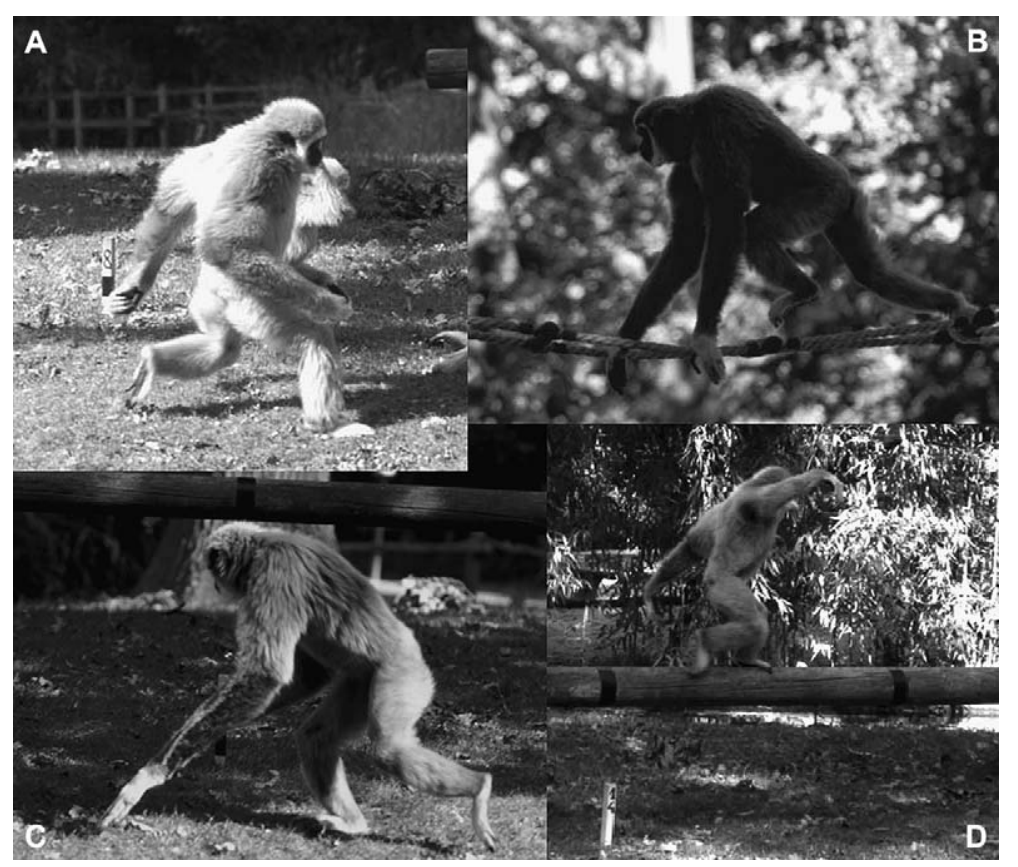

Fig. 5. Illustration of some gait types: (A) bipedal running, (B) arboreal quadrupedalism, (C) terrestrial quadrupedalism, and (D) bipedal walking on pole. 
Table 3

Mean (X), standard deviation (STDEV), and minimal and maximal values (RANGE) for the spatiotemporal parameters of the hind limbs during the different gait types

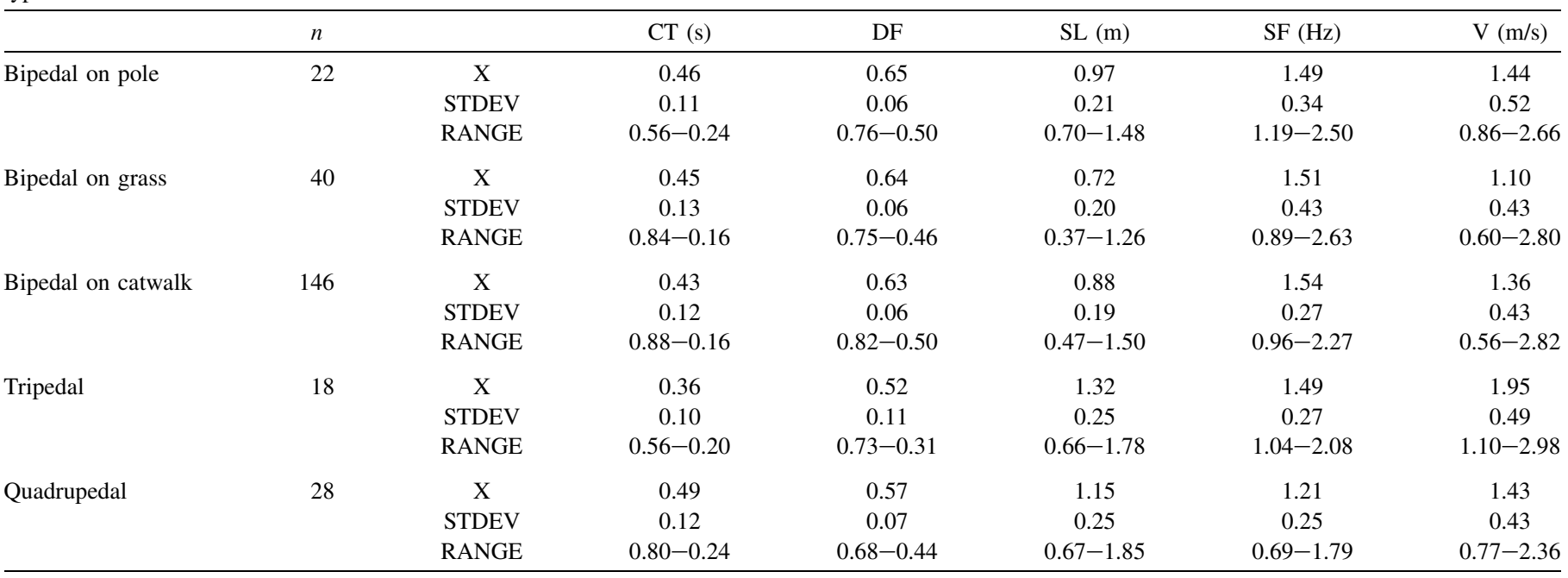

Abbreviations as in Table 2.

analyzed sequences for each gait type cover a large range of speeds, with a correspondingly wide range of spatiotemporal parameters. There is a significant correlation between dimensionless velocity and the DF, DSL, and DSF for each gait type, and linear equations of these relationships could be calculated (Table 4). The statistically significant differences in slopes and intercepts between the different gait types are presented in Table 5.

Judging from the regression plots in Figure 6, it appears that the different gait types are not very distinct from each other in their hind limb spatiotemporal aspects. A detailed description of the effect of velocity on the spatiotemporal parameters is given below for each gait type.

\section{Bipedalism (s.s.)}

We calculated separate regression lines for the three conditions - the pole, the grass, and the catwalk-for bipedal walking (Table 4). Bipedal running, galloping, and hand-assisted bipedal walking were excluded from the analysis. There are no differences in slope between the regression lines of DSL and DSF with DV during the different bipedal conditions

Table 4

Linear regression equations between the spatiotemporal parameters of the hind limbs and dimensionless walking velocity (DV) for each gait type

\begin{tabular}{lccc}
\hline & DF & DSL & DSF \\
\hline $\begin{array}{l}\text { Bipedal on } \\
\text { pole }\end{array}$ & $-0.09 \mathrm{DV}+0.76^{* * *}$ & $2.57 \mathrm{DV}+3.09^{* * *}$ & $0.07 \mathrm{DV}+0.11^{* * *}$ \\
$\begin{array}{l}\text { Bipedal on } \\
\text { grass }\end{array}$ & $-0.11 \mathrm{DV}+0.74 * * *$ & $2.83 \mathrm{DV}+2.45^{* * * *}$ & $0.07 \mathrm{DV}+0.11^{* * *}$ \\
$\begin{array}{l}\text { Bipedal on } \\
\text { catwalk }\end{array}$ & $-0.15 \mathrm{DV}+0.79 * *$ & $2.55 \mathrm{DV}+2.57 * * *$ & $0.09 \mathrm{DV}+0.10^{* * *}$ \\
$\begin{array}{l}\text { Tripedal } \\
\text { Quadrupedal }\end{array}$ & $-0.17 \mathrm{DV}+0.80^{* *}$ & $2.77 \mathrm{DV}+3.61^{* *}$ & $0.05 \mathrm{DV}+0.12^{* *}$ \\
\end{tabular}

Significance levels: *p $<0.05, * * \mathrm{p}<0.01, * * * \mathrm{p}<0.001$.

Abbreviations as in Table 2. $(p>0.05)$. However, there is a difference in intercept between the DSL regression lines for the pole and catwalk conditions ( $\mathrm{p}<0.01$; Table 5), with significantly larger DSLs occurring on the pole (Fig. 6, Table 4). The DF decreases more rapidly with increasing DV during bipedalism on the catwalk than it does on the pole (significantly different slope; $p<0.05$ ), but the slopes of the DF regression lines (as a function of DV) are similar for the other bipedal conditions $(\mathrm{p}>0.05$; Table $5)$. The spatiotemporal gait parameters for bipedal pole-walking are comparable to those for bipedal walking on grass, but differ slightly from those on the catwalk (DF slope and DSL intercept; $\mathrm{p}<0.05)$. The gait characteristics for bipedalism are statistically similar on both substrates.

Comparison of the bipedal sequences with the tripedal and quadrupedal sequences (Table 5) reveals that the slopes of the regression lines for DSL, DSF, and DF as a function of DV are

Table 5

Matrix representation of the Tukey post-hoc test, showing the significant differences in slope (in italic) and/or intercept (between brackets) between the hind limb spatiotemporal parameters of the different gait types $(\mathrm{p}<0.05)$

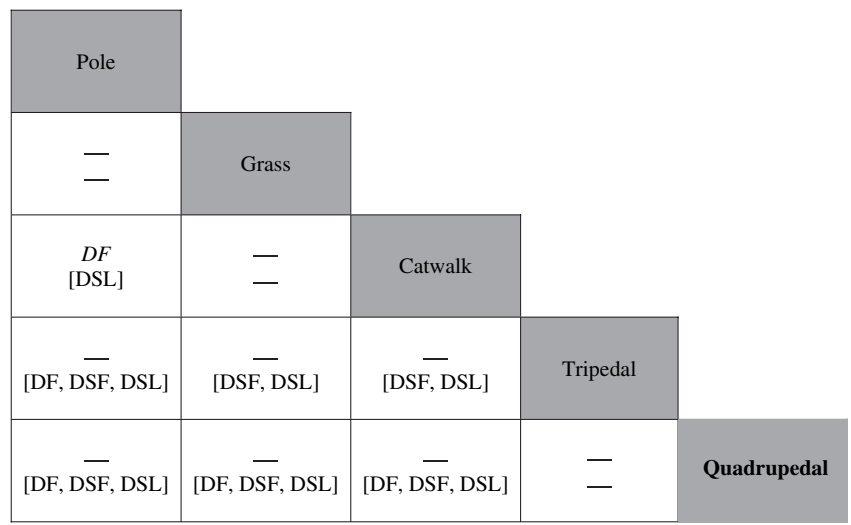

Abbreviations as in Table 2. 

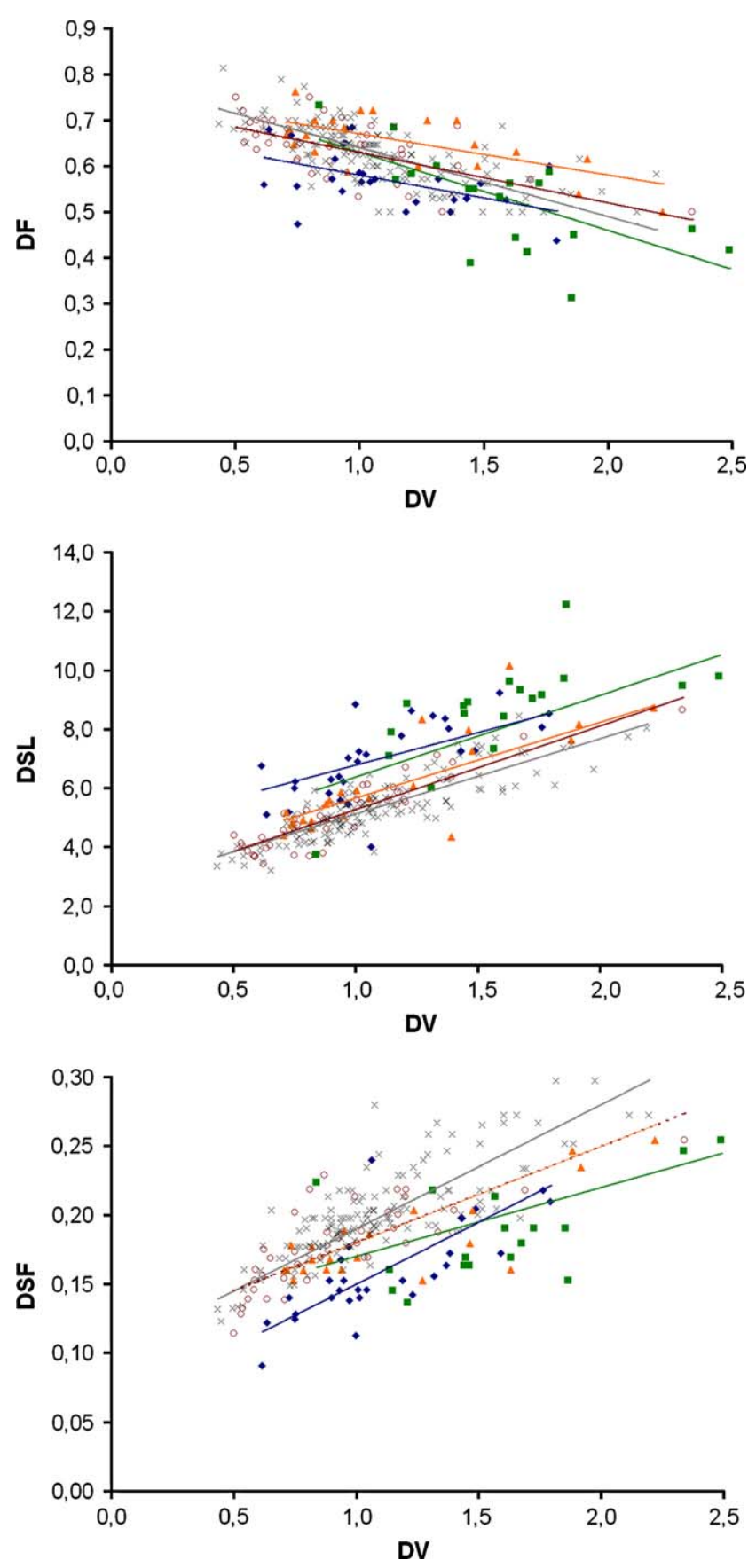

Fig. 6. Scatter plot of duty factor (DF; top), dimensionless stride length (DSL; middle), and dimensionless stride frequency (DSF; bottom) of the hind limbs as a function of dimensionless walking velocity (DV). A linear trend line is drawn for each gait type, and the corresponding equations are given in Table 4. Legend: bipedal on grass $=$ red circle, bipedal on pole $=$ orange triangle, bipedal on catwalk $=$ grey cross, tripedal $=$ green square, and quadrupedal $=$ blue diamond.

similar for the different gait types. Differences between the gait types are mainly situated in differences in intercepts of the regression lines. The DF and DSF are higher during bipedal walking-on the pole, on grass, and on the catwalk - than during quadrupedalism $(\mathrm{p}<0.001)$, but DSL is larger during quadrupedalism $(\mathrm{p}<0.01)$ and tripedalism $(\mathrm{p}<0.05)$ than during all bipedal walking gaits $(\mathrm{p}<0.0001)$.
We refer the reader to Vereecke et al. (2005b; see Table 2) for a full description of hylobatid bipedalism on the catwalk, including the effect of velocity changes on kinetics, plantar pressure, and spatiotemporal gait parameters.

\section{Tripedalism}

The spatiotemporal characteristics of the hind limbs during tripedal galloping and tripedal skipping are statistically similar and, therefore, both gait types were combined into a common tripedal gait class for the analyses. The hind limb gait characteristics of tripedalism are slightly different from those of bipedalism and quadrupedalism, which is reflected in the regression lines for the different spatiotemporal gait parameters (Fig. 6). The slopes for the tripedal DSF, DF, and DSL regression lines (as a function of DV) are similar to those for the other gait types $(\mathrm{p}>0.05)$, but differences are found in the intercepts of the regression lines. The DF is lower during tripedalism than during bipedalism on the pole $(\mathrm{p}<0.05)$ but similar to the DF for bipedalism on the catwalk and on grass $(\mathrm{p}>0.05)$. The DSF is lower and the DSL is larger during tripedalism than during all bipedal gaits - on grass, pole, and catwalk $(\mathrm{p}<0.001)$. There are no significant differences between the spatiotemporal parameters of tripedalism and quadrupedalism ( $\mathrm{p}>0.05$; Table 5).

The correlation between the spatiotemporal parameters of the fore- and hind limbs during tripedalism is significant for CT $(n=13, \mathrm{p}<0.01)$, the SF $(n=12, \mathrm{p}<0.01)$, and the SL ( $n=11, \mathrm{p}<0.0001)$, but there is not a significant correlation between the duty factors of the fore- and hind limbs ( $n=12 ; \mathrm{p}>0.05$; Fig. 7). The stride lengths of the forelimbs are approximately twice as long as the stride lengths of the hind limbs, and the stride frequencies of the hind limbs are roughly double that of the forelimbs. This is due to the fact that there are two (leading) hind limb cycles for each forelimb cycle. The CT of the forelimbs is slightly longer than the CT of the hind limbs. The forelimbs appear to have a rather symmetrical and regular gait cycle, with contact of the opposite hand occurring at $50 \%$ of the cycle duration. Thus, the forelimb stride duration (i.e., the time between two subsequent contacts of the same hand) is equal to twice the forelimb step duration (i.e., the time between contact of left and right hand). There is a significant correlation between each of the forelimbs spatiotemporal gait characteristics and velocity $(\mathrm{r}>0.6, \mathrm{p}<0.05$; $n=13)$. The CT and DF of the forelimbs increase and the SL and SF decrease with increasing velocity.

\section{Quadrupedalism}

For the statistical analyses, we combined the crutching walk and the half-bound into a common quadrupedal gait class since the spatiotemporal characteristics of their hind limbs appear to be statistically similar. This allowed us to make a comparison between the quadrupedal, bipedal, and tripedal gait types. Quadrupedalism has somewhat different hind limb gait characteristics than bipedalism, as can be seen in the regression plots (Fig. 6). The quadrupedal and bipedal regression lines for DF, DSL, and DSF as a function of DV are parallel to each other, but there are significant differences in the intercepts. During 

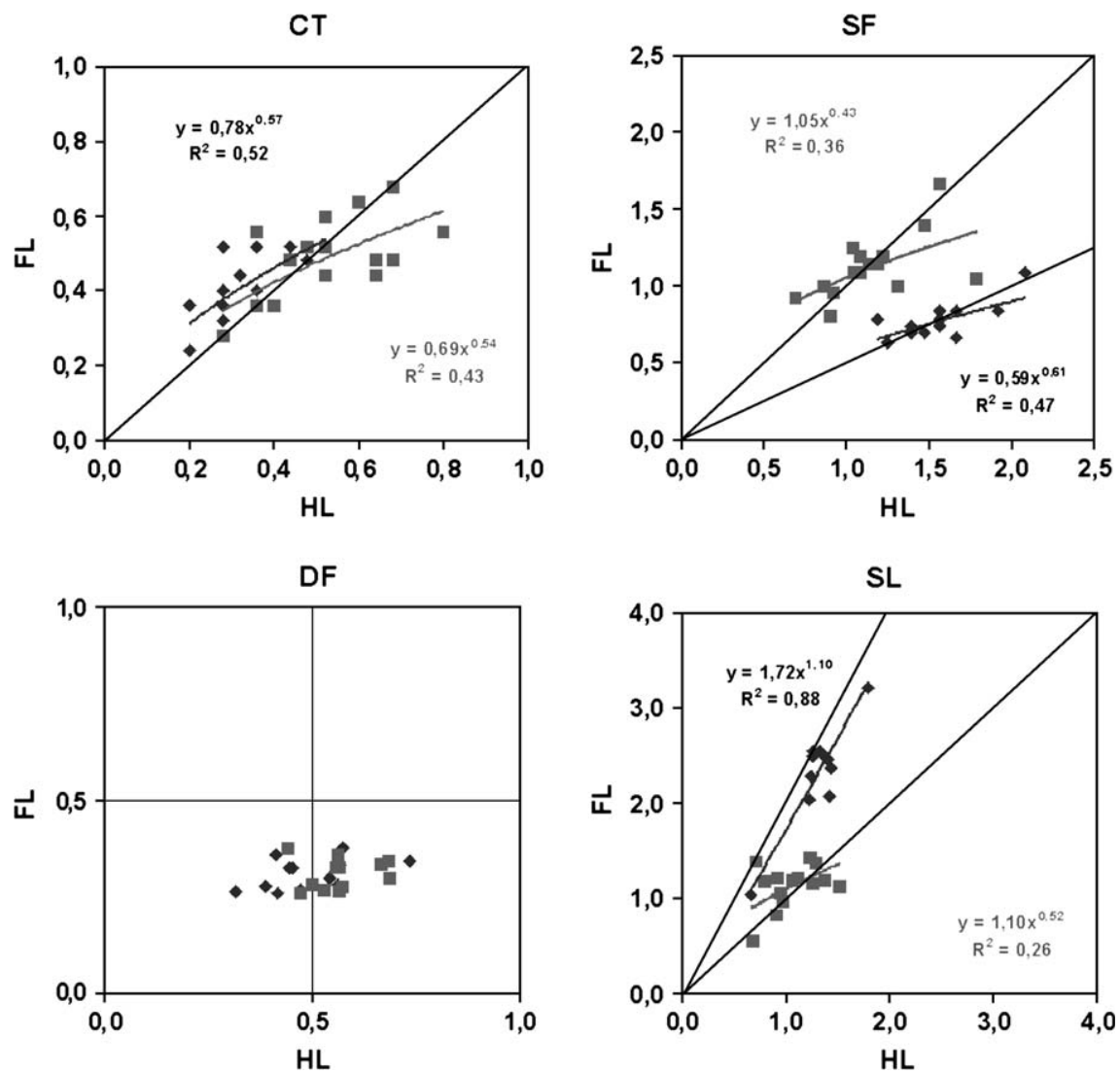

Fig. 7. Scatter plots of contact time (CT), stride frequency (SF), duty factor (DF), and stride length (SL) showing correlation between spatiotemporal parameters of fore- and hind limbs during tripedal (diamonds) and quadrupedal walking (squares) in gibbons. A power function is drawn and the corresponding equations are given for the gait parameters with a significant correlation between fore- and hind limbs $(\mathrm{p}<0.05)$.

quadrupedalism, the hind limbs have lower DF and DSF and larger DSL than during the bipedal gaits (on the pole, the grass, and the catwalk; $\mathrm{p}<0.001$ ). The regression lines for the DSF, $\mathrm{DF}$, and DSL of quadrupedalism and tripedalism have similar slopes and intercepts $(\mathrm{p}>0.05$; Table 5).

The correlation between the spatiotemporal parameters for the fore- and hind limbs during quadrupedalism is significant for CT $(n=15 ; \mathrm{p}<0.01)$, SF $(n=15 ; \mathrm{p}<0.01)$, and SL $(n=15 ; \mathrm{p}<0.05)$, but again, the correlation between the DF for the fore- and hind limbs is not significant $(n=15$; $\mathrm{p}>0.05$; Fig. 7). The spatiotemporal characteristics of the fore- and hind limbs are comparable, but the CT, SF, and SL of the forelimbs increase more slowly than those of the hind limbs (Fig. 7). There is a significant correlation between all of the spatiotemporal parameters of the forelimbs and velocity, but the correlation is rather weak for DF and SL $(r=0.49$ and 0.44 , respectively; $\mathrm{p}<0.05 ; n=15$ ) and strong for $\mathrm{CT}$ and $\mathrm{SF}$ $(\mathrm{r}=0.80 ; \mathrm{p}<0.0001 ; n=15)$.

\section{Comparison of gibbon, bonobo, and human bipedalism}

In Figure 8, we have added the gait parameters for human and bonobo bipedalism to the bipedal data from our gibbon sample, on grass and on the catwalk; the linear regression equations for DSL and DSF as a function of dimensionless walking speed of the three species are given in Table 6 . There are significant differences in slope between the DSL and DSF regression lines with DV of gibbon, bonobo, and human bipedalism $(\mathrm{p}<0.01)$. These differences are between human and gibbon bipedalism on grass for the DSL regression line $(\mathrm{p}<0.05)$ and between human and gibbon bipedalism on the catwalk for the DSF regression line $(\mathrm{p}<0.0001)$. The DSF increases more rapidly with increasing DV during human bipedalism than during gibbon bipedalism on the catwalk, and DSL increases more slowly with increasing DV during human bipedalism than during gibbon bipedalism on grass. The slopes and intercepts for the DSL and DSF regression lines are similar for bonobo and human bipedalism ( $p>0.05)$. The DSF is significantly smaller and DSL is significantly larger during gibbon bipedalism on grass and on the catwalk, respectively, than during bonobo or human bipedalism $(\mathrm{p}<0.0001)$. It seems that, at a given relative speed, bipedally walking gibbons take relatively larger strides at relatively lower frequencies than bipedally walking bonobos and humans.

\section{Discussion}

Terrestrial locomotion in gibbons and its implications for the evolution of hominin bipedalism

The data presented here constitute the first quantitative profile of terrestrial locomotion in gibbons. Baldwin and 

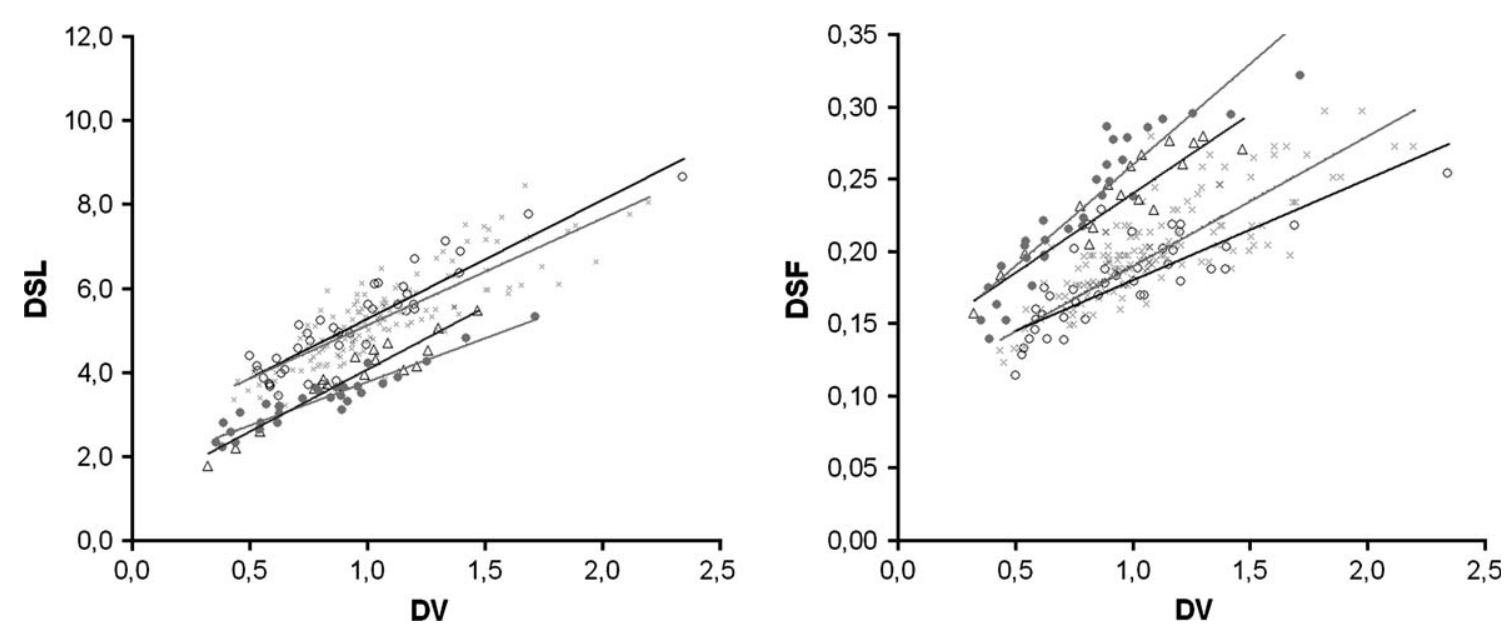

Fig. 8. Scatter plot of dimensionless stride length (DSL) and dimensionless stride frequency (DSF) for the hind limbs as a function of dimensionless walking velocity (DV) during bipedal walking in gibbons, bonobos, and humans. A linear regression line is drawn for each species, and the corresponding equations are given in Table 6. Legend: bipedal gibbon $($ grass) $=$ open circle, bipedal gibbon (catwalk) $=$ grey cross, bipedal bonobo $=$ triangle, and bipedal human $=$ grey circle.

Teleki (1976) reported on bipedalism, tripedalism, and quadrupedalism in a group of captive gibbons, but they only provided a qualitative description of these gait types. We were able to collect spatiotemporal parameters during spontaneous terrestrial locomotion of a group of white-handed gibbons $(H$. lar $)$, on several substrates and using different locomotor modes, and on this basis we identified some additional gait types that had not been reported earlier.

Most remarkable is the high diversity in locomotor modes of (captive) gibbons. We observed seven distinct gait types during terrestrial travel and these different gaits were used at a wide range of speeds by each of the animals, although there appears to be an individual preference for certain gaits. Gibbons seem to choose randomly which gait type they are going to use, and they switch smoothly from one gait type to another, even within one locomotor bout. Restricted speed ranges, in which certain gaits did or did not occur, and clear speed-related gait transitions were not detected (except for bipedal walking and running). Although speed does not seem to trigger specific gait types, it is likely that other behavioral, environmental, and/or physical factors (e.g., maximal strain and force) do (Bertram, 2005). We found significant inter-

Table 6

Slope, intercept, and significance level of the linear regression equations for dimensionless stride length (DSL) and frequency (DSF) of the hind limbs as a function of dimensionless walking velocity (DV) for gibbon, bonobo, and human bipedalism

\begin{tabular}{lccccc}
\hline Species & \multicolumn{2}{c}{ DSL } & & \multicolumn{2}{c}{ DSF } \\
\cline { 2 - 3 } \cline { 5 - 6 } & slope & intercept & & slope & intercept \\
\hline Gibbon on grass & $2.83^{* * a}$ & 2.45 & & $0.07 * * *$ & 0.11 \\
Gibbon on catwalk & $2.55^{* *}$ & 2.57 & & $0.09 * * * a$ & 0.10 \\
Bonobo & $2.95^{* *}$ & 1.12 & & $0.11^{* * *}$ & 0.13 \\
Human & $2.08^{*} *^{\mathrm{a}}$ & 1.70 & & $0.14 * * *^{\mathrm{a}}$ & 0.12 \\
\hline
\end{tabular}

Abbreviations and significance levels as in Tables 2 and 4 .

a The slopes of regression lines are significantly different between the species with the same superscript. individual variation in most gait parameters during the different gait types, even though we corrected for differences in body size. Part of the apparent inter-individual variation seems to be due to simple individual differences. However, as we worked with animals of different ages, we presume that age differences may also be an important source of variation.

Even though terrestrial locomotion is not included in the locomotor repertoire of wild gibbons, it appears that gibbons are not only skilled brachiators, but adept terrestrial locomotors as well. Carpenter (1964: 188) observed that "gibbons are superbly arboreal, inadequately terrestrial and almost completely helpless in water." We do not question his first and last statements, but we have some reservations about his opinion regarding the terrestrial locomotion of gibbons. Our observations lead us to conclude that gibbons are specialized for arboreal locomotion but that they are not limited to it. They are also adept in terrestrial locomotion, which is highly prevalent in captive animals. Apparently, the daily locomotor activities of captive gibbons contribute not only to the development of bipedality, as suggested by Yamazaki and Ishida (1984), but also improve the development of other terrestrial gaits.

In the context of hominin evolution, the observation that the highly arboreal gibbon is able to walk bipedally when on the ground is consistent with the "arboreal hypothesis" (Stern, 1975; Prost, 1980; Fleagle et al., 1981), insofar as arboreal locomotion could be pre-adaptive for hominin bipedalism. Even so, this does not preclude a terrestrial quadrupedal phase prior to bipedality in human evolution, as assumed by the "terrestrial hypothesis" (Gebo, 1996). It only suggests that bipedalism might have evolved in a versatile, small-bodied hominoid (see also Isler, 2003) and that additional selective pressures (e.g., environmental changes, energetic optimization, carrying behavior) might have lead to an increased amount of terrestrial travel and consequent improvement of bipedal locomotion. Yet, this remains a speculation that cannot be tested with available data. More importantly, however, as 
these data show, there is, to a certain extent, no need for structural specialization to walk bipedally (though bipedal efficiency may be low). The transition from an arboreal ancestral ape to a terrestrial, upright bipedal hominin might not be so hard to accomplish. This view is also supported by a recent study of bipedal and quadrupedal walking in bonobos (D'Août et al., 2004), which suggested that the ability to walk bipedally might be a mere "free bonus" locomotor mode. This also implies that the absence of obvious terrestrial or bipedal traits in hominin fossil remains does not necessarily preclude terrestrial travel and/or bipedalism, albeit non-habitual, as a component of their locomotor repertoire.

\section{Bipedal walking on different substrates}

Bipedal walking on the catwalk and on grass were compared because we wanted to know if gait parameters were affected by substrate type. In a previous study, we collected dynamic plantar pressure distributions and 3D ground reaction forces during bipedal walking on an instrumented catwalk, covered with a smooth surface (Vereecke et al., 2005b), and it is useful to know if the spatiotemporal parameters of these bouts corresponded with those of bipedal walking on the grassy island. No statistical differences were found between the gait parameters for both substrates, and we can therefore conclude that the spatiotemporal characteristics of the bipedal bouts collected on the catwalk are representative for bipedal walking in gibbons, irrespective of the substrate type used. There were, however, some differences between bipedalism on the pole and on the catwalk, whereas no differences were detected between the pole and grass conditions. This suggests that small differences do exist between the grassy and smooth substrate, but a marked effect of substrate type on bipedal gait characteristics is clearly absent.

During previous kinetic (Kimura, 1985) and kinematic analyses (Yamazaki and Ishida, 1984) of level versus (horizontal) pole-walking, only minor differences between arboreal ("pole") and terrestrial bipedalism were detected. The trunk is more inclined during pole-walking ( $33^{\circ}$ vs. $23^{\circ}$ with vertical; Yamazaki and Ishida, 1984), but the foot-force patterns of bipedal pole-walking are basically the same as on the floor. In line with this, we did not find major differences in the spatiotemporal characteristics of arboreal ("pole") and terrestrial bipedalism ("catwalk" and "grass") in gibbons. The relationships between the different spatiotemporal parameters and dimensionless velocity, as well as the average values of the gait parameters, were similar during bipedal walking on the pole and on grass. Some differences were found between bipedal walking on the pole and on the catwalk. The DF decreased less rapidly with increasing DV during bipedal walking on the pole compared to bipedal walking on the catwalk, and DSLs were slightly higher during bipedalism on the pole compared to bipedalism on the catwalk, although no corresponding statistically significant differences in DSFs was found (Fig. 6). Apparently, gibbons try to maintain relatively high duty factors and use relatively larger stride lengths (and slightly lower stride frequencies) during bipedal pole-walking.
High duty factors are associated with relatively long contact times, and large stride lengths entail larger limb angular excursions (Demes et al., 1990), which are both characteristic of compliant walking (Schmitt, 1999). This might be an adaptation for arboreal locomotion, as a compliant gait might improve balance (by providing a longer time to hold on) and can reduce potentially dangerous branch oscillations (by lowering stride frequency; Demes et al., 1990; Schmitt, 1999).

\section{Gibbon tripedalism and quadrupedalism}

It could be argued that the tripedal gait of gibbons is, in fact, a quadrupedal gait, as the four limbs contact the substrate during a gait cycle. However, since the fore- and hind limbs have a different stride frequency-each hand contact is followed by contact of both feet - there are only three supporting limbs during a complete hind limb cycle. To our knowledge, no gait with a similar footfall pattern has been described previously, which makes the tripedal gait of gibbons exceptional. Rhythmically, it resembles arm-swinging, with an alternating forward motion of the forelimbs and a coupled movement of both hind limbs. This might suggest that a similar neural control is used during tripedalism and brachiation. The forces acting on the limbs are, however, different between these gait types, as brachiation causes tension (Bertram, 2004) and tripedalism leads to compression of the forelimb joints.

The quadrupedal gait of gibbons is also remarkable because the footfall patterns are more comparable to the bounding of some old world monkeys (e.g., colobines and langurs; McGraw, 1996; Byron and Covert, 2004; Workman and Covert, 2005) and the half-bounding of medium-sized mammals (e.g., rabbits, chinchillas, and bandicoots; Fischer et al., 2002; Lammers and German, 2002; Bennett and Garden, 2004) than to the typical diagonal sequence gait of most primates (Hildebrand, 1967; Vilensky, 1989; Larson, 1998; Lemelin et al., 2003).

In the half-bound of mammals, the forelimbs touchdown successively, followed by a synchronized forward movement of both hind limbs, whereas in gibbons, both forelimbs touchdown simultaneously, followed by a successive touchdown of the hind limbs. In the bounding gait of several Old World monkeys, which is commonly used during locomotion on arboreal substrates (McGraw, 1996; Byron and Covert, 2004), both forelimbs touch down simultaneously, followed by simultaneous touchdown of both hind limbs.

Despite its remarkable characteristics and common occurrence in captivity, hylobatid quadrupedalism has only been sporadically described. One of the first descriptions of quadrupedalism in captive gibbons was given by Carpenter (1964). He noticed the simultaneous arm movements and the palmigrade hand contact but ultimately compared gibbon quadrupedalism with chimpanzee knuckle-walking. Hylobatid quadrupedalism is, however, clearly distinct from chimpanzee (or bonobo) knuckle-walking. Chimpanzee knuckle-walking is a diagonal sequence or forward cross-type gait (i.e., right hind limb, left forelimb, LH, RF; Hildebrand, 1967; Tuttle, 1970; Kimura, 1985; Reynolds, 1987), with the hands contacting the substrate with the knuckles, whereas gibbon quadrupedalism 
has simultaneous arm movements (RH, LH, R-LF) and the knuckles do not contact the substrate (Baldwin and Teleki, 1976; pers. obs.). Hrdlička (1940) also observed quadrupedally walking gibbons but noted that they walked on their knuckles. Together with the brief report of Hildebrand (1967) and the more detailed description of Baldwin and Teleki (1976), these are the only reports of hylobatid quadrupedalism that we have found in the literature, and several other researchers have even postulated that gibbons are not capable of walking quadrupedally (Hollihn, 1984; Isler, 2005). To date, we have found no records of quadrupedalism in the wild, but from time to time, we did observe our captive gibbons walking quadrupedally on branches and on rope ladders (Fig. 4B), which suggests that quadrupedalism might also be used during arboreal travel in wild gibbons.

A related gait type that has been reported repeatedly-both in the wild and in captivity-is quadrumanous climbing (Baldwin and Teleki, 1976; Fleagle, 1976; Yamazaki and Ishida, 1984; Cannon and Leighton, 1994; Sati and Alfred, 2002; Isler, 2002, 2003, 2005). Gibbons are very able vertical climbers (Isler, 2002, 2005), and on slightly inclined supports, wild siamangs (Symphalangus syndactylus) have been reported to shift from climbing to a regular quadrupedal gait (Fleagle, 1976). During an experiment with a changeable substrate inclination, a white-handed gibbon was also observed to walk quadrupedally up to an inclination of $40^{\circ}$ (Nakano, 2002). Unfortunately, footfall patterns were not described on horizontal or inclined substrates, but we assume the gait is different from the half-bound or crutching walk that we observed during terrestrial travel. Isler (2002) provided footfall patterns for vertical climbing in gibbons and concluded that gibbons use all possible footfall patterns, $17.7 \%$ of which are asymmetrical, in contrast to the quite homogenous footfall patterns of other primates. Isler (2005) attributed these irregular footfall patterns and pronounced differences between fore- and hind limb gait parameters to a deficient quadrupedal limb coordination in gibbons. However, the observation that gibbons possess a very diverse locomotor repertoire and do locomote quadrupedally regularly leads us to question this statement. A more plausible reason for the atypical crutching gait in gibbons might be their exceptionally long forelimbs, amounting to up to $165 \%$ of the lower limb length (Schultz, 1973). These body proportions are advantageous during brachiation but might hamper a regular diagonal sequence gait due to interference of fore- and hind limbs.

The spatiotemporal characteristics of the tripedal and quadrupedal gaits respond to changes in velocity in a way that is rather similar to those of bipedal walking. The gait parameters for each gait type are strongly correlated with velocity, and there are only minor differences between the distinct gait types (cf. bonobos; Aerts et al., 2000). The stride lengths are relatively longer during tripedalism and quadrupedalism compared to bipedalism, which can be explained by the presence of a hind limb aerial phase during both gait types. The duty factors are also relatively lower during quadrupedalism, resulting from lengthened absolute stride durations, which can again be ascribed to the presence of a flight phase.

\section{Comparison of gibbon, bonobo, and human bipedalism}

We compared gibbon bipedalism with bonobo and human bipedalism because we wanted to know how the bipedal locomotion of gibbons, which have a distinct morphology and seem to lack any bipedal specializations, compares to the bipedal locomotion of bonobos and humans. Bonobos, along with chimpanzees, are the closest living relatives of humans (Chen and $\mathrm{Li}$, 2001; D'Août et al., 2004; Goodman et al., 2005), and they occasionally walk bipedally (Susman et al., 1980), which makes them suitable for the study of the evolution of human bipedalism. In addition, it has been suggested that the postcranial morphology of bonobos shows the closest affinity with the morphology of the Pan-hominin ancestor (Zihlman and Cramer, 1978; McHenry and Corruccini, 1981; Payne, 2001).

We found some marked differences when we compared the spatiotemporal gait parameters of bipedal walking for gibbons, bonobos, and humans. Gibbons, bonobos, and humans increase speed by increasing both stride frequency and length, but at a given relative speed, gibbons tend to take relatively larger strides at relatively lower frequencies than either bonobos or humans. A possible explanation for these relatively low stride frequencies might be found in the natural pendular periods (NPPs) of the gibbon hind limb. The NPP is the amount of time it takes the limb to swing through one complete oscillation if it swings as a true pendulum (Raichlen, 2004b). Arboreality has been linked with heavier distal limb segments (Raichlen, 2004a), and Myers and Steudel (1997) suggested that relatively distal limb mass distributions should increase a limb's NPP. We might, therefore, expect that the highly arboreal gibbon has more distally distributed limb masses, resulting in larger NPPs, lower (dimensionless) stride frequencies and longer (dimensionless) stride lengths (Raichlen, 2004b), which, in turn, are associated with increased limb angular excursions (Demes et al., 1990). Using low stride frequencies and large stride lengths might also be an arboreal adaptation to reduce potentially dangerous branch oscillations during arboreal travel (Demes et al., 1990). In addition, maintaining low stride frequencies (or high CT and DF) might reduce the energetic cost of travel, as an increase in stride frequency almost matches the increase in rate of energy consumption per kilogram per stride (Heglund and Taylor, 1988; Roberts et al., 1998; Bramble and Lieberman, 2004; Doke et al., 2005).

Bipedal walking appears to be relatively fast in gibbons compared to that of bonobos and humans, and gibbons could keep on walking at speeds at which humans have to start running. A human with a hind limb length of $0.9 \mathrm{~m}$ will shift from walking to running at approximately $2.0-2.5 \mathrm{~m} / \mathrm{s}$, as predicted by the inverted pendulum principle $[\mathrm{V}<\sqrt{ }(\mathrm{g} \times 0.9)$; Alexander, 1992]. In gibbons and other primates, the walk-run transition is delayed because of their compliant bipedal gait (Ishida et al., 1976; Schmitt, 1999), and an inverted pendulum mechanism is probably absent. We observed gibbons "walking" bipedally at speeds up to $2.8 \mathrm{~m} / \mathrm{s}$, which are very fast "walking" speeds considering that the gibbon hind limb is approximately half as long as the human hind limb. However, the kinematics of these high-speed "walking" bouts are 
comparable to running sequences. Whether this is walking or running is a matter of definition. ${ }^{2}$ It would probably be better to talk about locomotion with and without an aerial phase to circumvent discussion of this point (e.g., see discussion of grounded vs. aerial running in the ostrich; Rubenson et al., 2004).

According to the spatiotemporal gait parameters, there is a greater resemblance between bonobo and human bipedalism than between gibbon and human bipedalism. Gibbons and bonobos are both highly arboreal apes that occasionally walk bipedally in the wild. The observed differences in spatiotemporal characteristics are thus probably related to the distinct morphology and/or the more distant phylogenetic position of gibbons in comparison with bonobos and humans.

\section{Conclusion}

The most remarkable result of this study is the high diversity of locomotor modes in (captive) gibbons. We observed seven distinct gait types during terrestrial travel, and these different gaits were used at a wide range of speeds by each of the animals. Obviously, adaptations for arboreality and brachiation, such as the specialized body proportions, have not constrained the gibbon's terrestrial locomotor abilities. In addition, this might imply that the step from an arboreal ancestral ape to a terrestrial upright bipedal hominin might not have been difficult to accomplish and that structural specializations are not a prerequisite for the adoption of a (non-habitual) bipedal gait.

Comparison of the spatiotemporal characteristics of bipedal walking on the different substrates (grass, smooth surface) revealed that substrate type has only a minor influence on the bipedal gait characteristics of gibbons. Pole-walking is slightly different from terrestrial (grass and catwalk) bipedalism: the duty factors decrease more slowly with increasing dimensionless velocity on the pole than on the catwalk, and the dimensionless stride lengths are slightly higher during bipedal pole-walking. Both differences are associated with a compliant gait, which might improve balance during arboreal locomotion. The spatiotemporal characteristics of the tripedal and quadrupedal gaits are comparable to those of bipedal walking, but the footfall patterns are exceptional and are unlike those of any other primate gait.

Gibbons, bonobos, and humans increase speed by increasing both stride frequency and length, but DSF increases more rapidly with DV during human bipedalism than during gibbon bipedalism on the catwalk. Moreover, at a given speed, gibbons tend to take relatively larger strides at lower frequencies than bonobos and humans. Bipedal walking also appears to be relatively fast in gibbons compared to that of bonobos and humans. Gibbons walked at speeds at which humans have to start running. According to the spatiotemporal gait parameters, there is a greater resemblance between bonobo and human bipedalism than between gibbon and human bipedalism.

\footnotetext{
${ }^{2}$ Refer to Footnote 1.
}

\section{Acknowledgements}

This study was supported by a research grant to E.E.V. and research project funding to P.A. (G.0209.99) from the Fund for Scientific Research, Flanders (Belgium). We thank the Flemish Government for structural support to the Centre for Research and Conservation of the Royal Zoological Society of Antwerp. We are also grateful to Professor Daniel Schmitt and two anonymous referees for their helpful comments on the original manuscript. Finally, we also thank the very cooperative staff of the Wild Animal Park Planckendael, Belgium.

\section{References}

Adachi, K., Nishizawa, S., Endo, B., 1996. The trajectory of the point of application of the resultant force of body mass at different walking speeds; statistical analysis of human walking. Folia Primatol. 66, 160-180.

Aerts, P., Van Damme, R., Van Elsacker, L., Duchene, V., 2000. Spatio-temporal gait characteristics of the hind limb cycles during voluntary bipedal and quadrupedal walking in bonobos (Pan paniscus). Am. J. Phys. Anthropol. 111, 503-517.

Alexander, R.M., 1977. Mechanics and scaling of terrestrial locomotion. In: Pedley, T.L. (Ed.), Scale Effects in Animal Locomotion. Academic Press, London, pp. 93-110.

Alexander, R.M., 1982. Walking, running and jumping. In: Alexander, R.M. (Ed.), Locomotion of Animals. Blackie, Glasgow, pp. 81-113.

Alexander, R.M., 1992. Exploring Biomechanics. Animals in Motion. Scientific American Library, New York.

Alexander, R.M., 2004. Bipedal animals, and their differences from humans. J. Anat. 204, 321-330.

Alexander, R.M., Jayes, A.S., 1983. A dynamic similarity hypothesis for the gaits of quadrupedal mammals. J. Zool. 201, 135-152.

Andrew, P., Groves, C.E., 1976. Gibbon and brachiation. In: Rumbaugh, D.M. (Ed.), Suspensory Behavior, Locomotion, and Other Behaviors of Captive Gibbons: Cognition. Gibbon and Siamang: A Series of Volumes on the Lesser Apes, vol. 4. Karger, Basel, pp. 167-218.

Baldwin, L.A., Teleki, G., 1976. Patterns of gibbon behavior on Hall's Island, Bermuda: a preliminary ethogram for Hylobates lar. In: Rumbaugh, D.M. (Ed.), Suspensory Behavior, Locomotion, and Other Behaviors of Captive Gibbons: Cognition. Gibbon and Siamang: A Series of Volumes on the Lesser Apes, vol. 4. Karger, Basel, pp. 21-105.

Bennett, M.B., Garden, J.G., 2004. Locomotion and gaits of the northern brown bandicoot, Isoodon macrourus (Marsupalia: Peramelidae). J. Mammal. 85, 296-301.

Bertram, J.E.A., Chang, Y.H., 1996. Gait change in gibbon brachiation. Am. Zool. 36, 20A.

Bertram, J.E.A., Ruina, A., Cannon, C.E., Hui Chang, Y., Coleman, M.J., 1999. A point-mass model of gibbon locomotion. J. Exp. Biol. 202, 2609-2617.

Bertram, J.E.A., Chang, Y.H., 2001. Mechanical energy oscillations of two brachiation gaits: measurement and simulation. Am. J. Phys. Anthropol. 115, 319-326.

Bertram, J.E.A., 2004. New perspectives on brachiation mechanics. Yearb. Phys. Anthropol. 47, 100-117.

Bertram, J.E.A., 2005. Constrained optimization in human walking: cost minimization and gait plasticity. J. Exp. Biol. 208, 979-991.

Bramble, D.M., Lieberman, D.E., 2004. Endurance running and the evolution of Homo. Nature 432, 345-352.

Byron, C.D., Covert, H.H., 2004. Unexpected locomotor behaviour: brachiation by an Old World monkey (Pygathrix nemaeus) from Vietnam. J. Zool. 263, 101-106.

Caldwell, G.E., Whitall, J., 1995. An energetic comparison of symmetrical and asymmetrical human gait. J. Mot. Behav. 27, 139-154. 
Cannon, C.H., Leighton, M., 1994. Comparative locomotor ecology of gibbons and macaques: selection of canopy elements for crossing gaps. Am. J. Phys. Anthropol. 93, 505-524.

Carpenter, C.R., 1964. A field study in Siam of the behavior and social relations of the gibbon (Hylobates lar). In: Carpenter, C.R. (Ed.), Naturalistic Behavior of Nonhuman Primates. The Pennsylvania State University Press, University Park, pp. 145-271.

Chang, Y.H., Bertram, J.E.A., Lee, D.V., 2000. External forces and torques generated by the brachiating white-handed gibbon (Hylobates lar). Am. J. Phys. Anthropol. 113, 201-216.

Chen, F.-C., Li, W.-H., 2001. Genomic divergences between humans and other hominoids and the effective population size of the common ancestor of humans and chimpanzees. Am. J. Hum. Genet. 68, 444-456.

D'Août, K., Aerts, P., De Clercq, D., De Meester, K., Van Elsacker, L., 2002. Segment and joint angles of hind limb during bipedal and quadrupedal walking of the bonobo (Pan paniscus). Am. J. Phys. Anthropol. 119, $37-51$.

D'Août, K., Vereecke, E., Schoonaert, K., De Clercq, D., Van Elsacker, L., Aerts, P., 2004. Locomotion in bonobos (Pan paniscus): differences and similarities between bipedal and quadrupedal terrestrial walking, and a comparison with other locomotor modes. J. Anat. 204, 353-361.

Demes, B., Jungers, W.L., Nieschalk, U., 1990. Size- and speed related aspects of quadrupedal walking in slender and slow lorises. In: Jouffroy, F.K., Stack, M.H., Niemitz, C. (Eds.), Gravity, Posture and Locomotion in Primates. Il Sedicesimo, Firenze, pp. 175-197.

Doke, J., Donelan, J.M., Kuo, A.D., 2005. Mechanics and energetics of swinging the human leg. J. Exp. Biol. 208, 439-445.

Ellefson, J.O., 1974. A natural history of gibbons in the Malayan peninsula. In: Rumbaugh, D.M. (Ed.), Gibbon and Siamang, vol. 3. Karger, Basel, pp. 1136.

Farley, C.T., Ferris, D.P., 1998. Biomechanics of walking and running: center of mass movements to muscle action. Exerc. Sport Sci. Rev. 26, 253-285.

Fischer, M.S., Schilling, N., Schmidt, M., Haarhaus, D., Witte, H., 2002. Basic limb kinematics of small therian mammals. J. Exp. Biol. 205, 1315-1338.

Fleagle, J.G., 1976. Locomotion and posture of the Malayan siamang and implications for hominoid evolution. Folia Primatol. 26, 245-269.

Fleagle, J.G., 1988. Primate Adaptation and Evolution. Academic Press, New York.

Fleagle, J.G., Stern, J.T., Jungers, W.L., Susman, R., 1981. Climbing: a biomechanical link with brachiation and with bipedalism. Symp. Zool. Soc. Lond. 48, 359-375.

Gebo, D.L., 1996. Climbing, brachiation, and terrestrial quadrupedalism: historical precursors of hominid bipedalism. Am. J. Phys. Anthropol. 101, 55-92.

Gittins, P.S., 1983. Use of forest canopy by the agile gibbon. Folia Primatol. 40, 134-144.

Goodman, M., 1999. Molecular Evolution '99: the genomic record of humankind's evolutionary roots. Am. J. Hum. Gen. 64, 31-39.

Goodman, M., Grossman, L.I., Wildman, D.E., 2005. Moving primate genomics beyond the chimpanzee genome. Trends Genet. 21, 511-517.

Heglund, N.C., Taylor, C.R., 1988. Speed, stride frequency and energy cost per stride: how do they change with body size and gait? J. Exp. Biol. 138, 301-318.

Hildebrand, M., 1967. Symmetrical gaits of primates. Am. J. Phys. Anthropol. 26, 119-130.

Hollihn, U., 1984. Bimanual suspensory behaviour: morphology, selective advantages and phylogeny. In: Preuschoft, H., Chivers, D.J., Brockelman, W.Y., Creel, N. (Eds.), The Lesser Apes: Evolutionary and Behavioural Biology. Edinburgh University Press, Edinburgh, pp. 85-95.

Hrdlička, A., 1940. Locomotion in gibbon. Am. J. Phys. Anthropol. 27, 481.

Ishida, H., Kimura, T., Okada, M., Yamazaki, N., 1976. Kinesiological aspects of bipedal walking in gibbons. In: Rumbaugh, D.M. (Ed.), Suspensory Behavior, Locomotion, and Other Behaviors of Captive Gibbons: Cognition. Gibbon and Siamang: A Series of Volumes on the Lesser Apes, vol. 4. Karger, Basel, pp. 135-145.

Ishida, H., Kimura, T., Okada, M., Yamazaki, N., 1984. Kinesiological aspects of bipedal walking in gibbons. In: Preuschoft, H., Chivers, D.J., Brockelman, W.Y., Creel, N. (Eds.), The Lesser Apes: Evolutionary and Behavioural Biology. Edinburgh University Press, Edinburgh, pp. 135-145.
Isler, K., 2002. Characteristics of vertical climbing in gibbons. Evol. Anthropol. 1 (Suppl.), 49-52.

Isler, K., 2003. 3D-kinematics of vertical climbing in hominoids. Ph.D. dissertation, Universität Zürich.

Isler, K., 2005. 3D-kinematics of vertical climbing in hominoids. Am. J. Phys. Anthropol. 126, 66-81.

Isler, K., Thorpe, S.K.S., 2003. Gait parameters in vertical climbing of captive, rehabilitant and wild Sumatran orang-utans (Pongo pygmaeus abelii). J. Exp. Biol. 206, 4081-4096.

Jungers, W.L., Stern, J.T., 1984. Kinesiological aspects of brachiation in lar gibbons. In: Preuschoft, H., Chivers, D.J., Brockelman, W.Y., Creel, N. (Eds.), The Lesser Apes: Evolutionary and Behavioural Biology. Edinburgh University Press, Edinburgh, pp. 119-134.

Kimura, T., 1985. Bipedal and quadrupedal locomotion of primates; comparative dynamics. In: Kondo, S. (Ed.), Primate Morphophysiology: Locomotor Analyses and Human Bipedalism. University of Tokyo Press, Tokyo, pp. 81-104.

Kowalchuk, R.K., Keselman, H.J., Algina, J., Wolfinger, R.D., 2004. The analysis of repeated measurements with mixed-model adjusted f-tests. Educ. Psychol. Meas. 64, 224-242.

Lammers, A.R., German, R.Z., 2002. Ontogenetic allometry in the locomotor skeleton of specialized half-bounding mammals. J. Zool. Lond. 258, 485495.

Larson, S.G., 1998. Unique aspects of quadrupedal locomotion in nonhuman primates. In: Strasser, E., Fleagle, J.G., Rosenberger, A.L., McHenry, H.M. (Eds.), Primate Locomotion: Recent Advances. Plenum Press, New York, pp. 157-173.

Lemelin, P., Schmitt, D., Cartmill, M., 2003. Footfall patterns and interlimb coordination in opossums (family Didelphidae): evidence for the evolution of diagonal-sequence walking gaits in primates. J. Zool. 260, 423-429.

McGraw, W.S., 1996. Cercopithecid Locomotion, Support Use and Support Availability in the Tai Forest, ivory Coast. Am. J. Phys. Anthropol. 110, 507-522.

McHenry, H.M., Corruccini, R.S., 1981. Pan paniscus and human evolution. Am. J. Phys. Anthropol. 54, 355-367.

Minetti, A.E., 1998. The biomechanics of skipping gaits: a third locomotion paradigm? Proc. R. Soc. Lond. B 265, 1227-1235.

Myers, M.J., Steudel, K., 1997. Morphological conservation of limb natural pendular period in the domestic dog (Canis familiaris): implications for locomotor energetics. J. Morphol. 234, 183-196.

Nakano, Y., 2002. The effects of substratum inclination on locomotor patterns in primates. Z. Morphol. Anthropol. 83, 189-199.

Okada, M., Yamazaki, N., Ishida, H., Kimura, T., Kondo, S., 1983. Biomechanical characteristics of hylobatid walking on flat surfaces. Ann. Sci. Nat. Zool. 5, 137-144.

Payne, R.C., 2001. Musculoskeletal adaptations for climbing in hominoids and their role as exaptations for the acquisition of bipedalism. Ph.D. thesis, University of Liverpool, Liverpool.

Peck, A.J., Turvey, M.T., 1997. Coordination dynamics of the bipedal galloping pattern. J. Mot. Behav. 29 (4), 311-325.

Preuschoft, H., Demes, B., 1984. Biomechanics of brachiation. In: Preuschoft, H., Chivers, D.J., Brockelman, W.Y., Creel, N. (Eds.), The Lesser Apes: Evolutionary and Behavioural Biology. Edinburgh University Press, Edinburgh, pp. 96-118.

Prost, J.H., 1967. Bipedalism of man and gibbon compared using estimates of joint motion. Am. J. Phys. Anthropol. 26, 135-148.

Prost, J.H., 1980. Origin of bipedalism. Am. J. Phys. Anthropol. 52, 175-189.

Raichlen, D.A., 2004a. Convergence of forelimb and hind limb natural pendular period in baboons (Papio cynocephalus) and its implication for the evolution of primate quadrupedalism. J. Hum. Evol. 46, 719-738.

Raichlen, D.A., 2004b. The relationship between limb muscle mass distribution and the mechanics and energetics of quadrupedalism in infant baboons (Papio cynocephalus). Ph.D. dissertation, University of Texas, Austin.

Reynolds, T.R., 1987. Stride length and its determinants in humans, early hominids, primates, and mammals. Am. J. Phys. Anthropol. 72, 101-115.

Roberts, T.J., Kram, R., Weyand, P.G., Taylor, R., 1998. Energetics of bipedal running. 1. Metabolic cost of generation force. J. Exp. Biol. 201, 2745-2751. 
Rubenson, J., Heliams, D.B., Lloyd, D.G., Fournier, P.A., 2004. Gait selection in the ostrich: mechanical and metabolic characteristics of walking and running with and without aerial phase. Proc. R. Soc. Lond. B 271, 1091-1099.

SAS Institute, Inc., 1999. SAS/STAT User's Guide, Version 7. SAS Institute, Inc., Cary, NC.

Sati, J.P., Alfred, J.R.B., 2002. Locomotion and posture in Hoolock gibbon. Ann. For. 10, 298-306.

Satterthwaite, F.E., 1941. Synthesis of variance. Psychometrika 6, 309-316.

Schaalje, G.B., McBride, J.B., Fellingham, G.W., 2001. Approximations to distributions of test statistics in complex mixed linear models using SAS ${ }^{\circledR}$ Proc MIXED. Paper 262-26 in the Proceedings of SAS ${ }^{\circledR}$ User Group International 26th Annual Conference, Long Beach, CA, April 22-25 2001.

Schmid, P., Piaget, A., 1994. Three-dimensional kinematics of bipedal locomotion. Z. Morphol. Anthropol. 80 (1), 79-87.

Schmitt, D., 1999. Compliant walking in primates. J. Zool. 247, 149-160.

Schultz, A.H., 1973. The skeleton of the Hylobatidae and other observations on their morphology. In: Rumbaugh, D.M. (Ed.), Gibbon and Siamang: A Series of Volumes on the Lesser Apes, vol. 2. Karger, Basel, pp. 1-54.

Susman, R.L., Badrian, N.L., Badrian, A.J., 1980. Locomotor behavior of Pan paniscus in Zaire. Am. J. Phys. Anthropol. 53, 69-80.

Stern, J.T., 1975. Before bipedality. Yearb. Phys. Anthropol. 19, 59-68.

Tuttle, R.H., 1970. Postural, propulsive, and prehensile capabilities in the cheiridia of chimpanzees and other great apes. In: Rumbaugh, D.M. (Ed.), The Chimpanzee, vol. 2. Karger, Basel, pp. 167-253.

Tuttle, R.H., 1972. Functional and evolutionary biology of hylobatid hands and feet. In: Rumbaugh, D.M. (Ed.), Gibbon and Siamang: A Series of Volumes on the Lesser Apes, vol. 1. Karger, Basel, pp. 136-206.

Tuttle, R.H., 1986. Positional behavior. In: Tuttle, R.H. (Ed.), Apes of the World: Their Social Behavior, Communication, Mentality, and Ecology. Noyes Publications, New Jersey, pp. 33-40.

Usherwood, J.R., Bertram, J.E.A., 2003. Understanding brachiation: insight from a collisional perspective. J. Exp. Biol. 206, 1631-1642.
Vereecke, E., D’Août, K., De Clercq, D., Van Elsacker, L., Aerts, P., 2003. Dynamic plantar pressure distribution during terrestrial locomotion of bonobos (Pan paniscus). Am. J. Phys. Anthropol. 120, 373-383.

Vereecke, E., D'Août, K., De Clercq, D., Van Elsacker, L., Aerts, P., 2004. The relationship between speed, contact time and peak plantar pressure in terrestrial walking of bonobos. Folia Primatol. 75, 266-278.

Vereecke, E., D'Août, K., Payne, R., Aerts, P., 2005a. Functional analysis of the foot and ankle myology of gibbons and bonobos. J. Anat. 206, 453-476.

Vereecke, E., D’Août, K., Van Elsacker, L., De Clercq, D., Aerts, P., 2005 b. Functional analysis of the gibbon foot during terrestrial bipedal walking: plantar pressure distributions and 3D ground reaction forces. Am. J. Phys. Anthropol. 128, 659-669.

Vilensky, J.A., 1989. Primate quadrupedalism: how and why does it differ from that of typical quadrupeds? Brain Behav. Evol. 34, 357-364.

Winter, D.A., 1991. The biomechanics and motor control of human gait: normal, elderly and pathological, second ed. University of Waterloo Press, Waterloo.

Whitall, J., Caldwell, G.E., 1992. Coordination of symmetrical and asymmetrical human gait. J. Mot. Behav. 24, 339-353.

Wolfinger, R.D., Chang, M., 1995. Comparing the SAS ${ }^{\circledR}$ GLM and MIXED procedures for repeated measures. In: Proceedings of the 20th Annual SAS User's Group International Conference. SAS Institute, Inc., Cary, NC, pp. 1172-1182.

Workman, C., Covert, H.H., 2005. Learning the Ropes: The Ontogeny of Locomotion in Red-Shanked Douc (Pygathrix nemaeus), Delacour's (Trachypithecus delacouri), and Hatinh Langurs (Trachypithecus hatinhensis) I. Positional Behavior. Am. J. Phys. Anthropol. 128 (2), 371-380.

Wunderlich, R.E., Schaum, J.C., Kilkenny, P.J., Sinopoli, M.C., 2004. Hind limb use during bipedalism in primates: a comparative study. J. Morphol. 206, 341.

Yamazaki, N., Ishida, H., 1984. A biomechanical study of vertical climbing and bipedal walking in gibbons. J. Hum. Evol. 13, 563-571.

Zihlman, A.L., Cramer, D.L., 1978. Skeletal differences between pygmy (Pan paniscus) and common chimpanzees (Pan troglodytes). Folia Primatol. 29, 86-94. 